\title{
Multidrug-resistant Neisseria gonorrhoeae failing treatment with ceftriaxone and doxycycline in France, November 2017
}

Thibault Poncin ${ }^{1,2}$, Sebastien Fouere ${ }^{4}$, Aymeric Braille ${ }^{1,2}$, Francois Camelena ${ }^{1,2,3}$, Myriem Agsous $s^{1,2}$, Cecile Bebear ${ }^{5}$, Sylvain

Kumanski ${ }^{2}$, Florence Lot ${ }^{6}$, Severine Mercier-Delarue ${ }^{1}$, Ndeindo Ndeikoundam Ngangro ${ }^{6}$, Maud Salmona ${ }^{1}$, Nathalie Schnepf ${ }^{1,2}$, Julie Timsitt, Magnus Unemo ${ }^{7}$, Beatrice Bercot ${ }^{1,2,3}$

1. Infectious Agents Department, Saint Louis Hospital, APHP, Paris, France

2. French National Reference Center for bacterial STI, Associated laboratory for gonococci, APHP, Paris, France

3. Paris Diderot University, IAME, UMR 1137, Sorbonne Paris Cité, Paris, France

4. Dermatology Department, CeGGID (Free Information Screening and Diagnostics Centers), Saint Louis Hospital, APHP, Paris, France

5. University of Bordeaux, USC EA 3671 Mycoplasmal and chlamydial infections in humans; Centre Hospitalier Universitaire de Bordeaux, French National Reference Center for bacterial STIs, Bordeaux, France

6. Santé Publique France, French National Public Health Agency, Saint-Maurice, France

7. WHO Collaborating Centre for Gonorrhoea and Other STIs, National Reference Laboratory for STIs, Department of Laboratory Medicine, Faculty of Medicine and Health, Örebro University, Örebro, Sweden

Correspondence: Beatrice Bercot (beatrice.bercot@lrb.aphp.fr)

We report a multidrug-resistant Neisseria gonorrhoeae urogenital and pharyngeal infection with ceftriaxone resistance and intermediate resistance to azithromycin in a heterosexual woman in her 205 in France. Treatment with ceftriaxone plus doxycycline failed for the pharyngeal localisation. Whole-genome sequencing of isolate $\mathrm{F}_{90}$ identified $\mathrm{MLST}_{1903}$, NG-MAST $\mathrm{ST}_{3435}$, NG-STAR ${ }_{233}$, and relevant resistance determinants. F90 showed phenotypic and genotypic similarities to an internationally spreading multidrugresistant and ceftriaxone-resistant clone detected in Japan and subsequently in Australia, Canada and Denmark.

The emergence of ceftriaxone resistance in Neisseria gonorrhoeae (NG) has caused public health concern worldwide [1-3] and it is a grave concern that the first global failure to treat pharyngeal gonorrhoea with dual antimicrobial therapy was reported in 2016 in the United Kingdom (UK) [4].

We report a multidrug-resistant (MDR) NG pharyngeal infection with ceftriaxone resistance and intermediate resistance to azithromycin that failed dual therapy with ceftriaxone and doxycycline. This treatment targeted an infection due to Chlamydiae trachomatis; the choice of the clinician was based on recent finding on the efficacy of doxycycline compared to that of azithromycin in urogenital infections [5].

\section{Case description}

In November 2017, a heterosexual woman in her early $20 s$ attended a local Hospital in Paris due to a vaginal discharge that had persisted for 3 days. She had only had unprotected sexual relations (oral and intercourse) with one regular French male partner who had urethritis symptoms in the 6 months prior and had received antimicrobial treatment at another clinic. Neither she nor her male partner had travelled abroad or had a known history of sexually transmitted infections. She was treated empirically with ceftriaxone ( $250 \mathrm{mg}$ intramuscularly) and doxycycline (100 mg orally twice a day, for 7 days). During the same visit, vaginal and pharyngeal swabs were sampled for detection of NG and Chlamydia trachomatis using nucleic acid amplification test (NAAT), RealTime CT/NG assay on the mzooo System, (Abbott Diagnosis, Abbott Molecular Inc., Des Plaines, IL, USA), and NG culture. NAAT and culture detected NG in both vaginal and pharyngeal samples, but all samples were negative for C. trachomatis. HIV, hepatitis B and C viruses, syphilis serological tests were also negative. At test of cure 4 weeks later, she had no symptoms or signs of gonorrhoea and was not given any additional treatment. However, it was found that only the vaginal swab was negative for NG, as the NAAT and culture taken from the pharyngeal swab remained positive, indicating that only the urogenital infection had been cured. Reinfection was excluded because the patient denied any sexual relations between the first visit and the test of cure. The patient and her male partner were 
requested to return for follow-up visit, but they are currently lost for follow-up.

\section{Microbiological investigation}

The NG test of cure isolate (referred to as F9o) was obtained on PolyVitex chocolate agar incubated at $+36 \pm 1{ }^{\circ} \mathrm{C}$ in a $5 \% \mathrm{CO}_{2}$-enriched atmosphere for 24 hours. F9o was investigated at the Associated laboratory for gonococci of the French National Reference Centre for bacterial STI, Paris, France, where Gramstained microscopy and matrix-assisted laser desorption/ionization-time of flight (MALDI-TOF) (Vitek MS, bioMérieux, Marcy l'Etoile, France) were used for NG species verification. Minimum inhibitory concentrations (MIC) of nine antimicrobials were determined by Etest (bioMérieux, Marcy l'Etoile, France) and interpreted using breakpoints from the European Committee on Antimicrobial Susceptibility Testing (EUCAST) [6].

F9o displayed resistance to ceftriaxone $(\mathrm{MIC}=0.5$ $\mathrm{mg} / \mathrm{L})$, cefixime $(M I C=1 \mathrm{mg} / \mathrm{L})$, tetracycline $(M I C=4$ $\mathrm{mg} / \mathrm{L}$ ), ciprofloxacin (MIC>32 $\mathrm{mg} / \mathrm{L}$ ), and rifampicin (MIC> $32 \mathrm{mg} / \mathrm{L}$ ). Furthermore, F9o showed intermediate resistance to azithromycin (MIC $=0.5 \mathrm{mg} / \mathrm{L})$, however, it was susceptible to spectinomycin (MIC $=8 \mathrm{mg} / \mathrm{L}$ ) and had low MIC for ertapenem $(\mathrm{MIC}=0.004 \mathrm{mg} / \mathrm{L})$ and gentamicin $(\mathrm{MIC}=8 \mathrm{mg} / \mathrm{L})$, for which EUCAST does not state resistance breakpoints.

\section{Molecular investigation}

Whole-genome sequencing (WGS) was performed in the Associated laboratory for gonococci of the French National Reference Centre for bacterial STI, Paris, France. DNA extraction was conducted using Wizard Genomic DNA Purification kit (Promega), as previously described [7]. Multiplexed DNA libraries were prepared with the Nextera XT construction protocol (Illumina, San Diego, CA, USA). Paired-end, 1.547.414 150-bp indexed reads with an average depth of 151 were obtained on a MiSeq platform (Illumina). De novo assembly was performed using SPAdes 3.11.1 software [8]. QUAST software [9] revealed that the assembly provided 106 contigs with an average length of 20,394 nucleotides, a $\mathrm{N}_{50}$ of 47,830 nucleotides. The contigs covered $93.7 \%$ of the genome of NG reference strain FA109o. The genome annotation was performed using the MicroScope platform (http://www.genoscope.cns.fr/ $\mathrm{agc} / \mathrm{microscope}$ ) [10]. The whole nucleotide sequence of the penA gene of Fgo is available on GenBank under accession number $\mathrm{MH} 172152$.

Using the WGS data, NG antimicrobial resistance determinants were determined in silico with the NG FA1090 genome as reference. Sequence types (ST) were also determined in silico from the WGS data using the NG multi-antigen sequence typing (NG-MAST) online database (http://www.ng-mast.net) [11], the multilocus sequence typing (MLST) from the PubMLST database [12], and the NG Sequence Typing for Antimicrobial Resistance (NG-STAR) [13].
F9o was assigned as $\mathrm{MLST}_{1903}$, NG-MAST $\mathrm{ST}_{3435}$, and NG-STAR ${ }_{233}$. Regarding ceftriaxone resistance determinants, the isolate harboured a mosaic penA-60.001 allele, sharing a $100 \%$ homology with the $p e n A_{\mathrm{FC}_{428}}$ of the Japanese ceftriaxone-resistant FC428 strain [14]. The $p e n A_{\mathrm{FC} 428}$ gene encodes a mosaic penicillin-binding protein $2\left(\mathrm{PBP}_{2}\right)$ including the amino acid alterations A311V, I312M, V316T, T483S, and G545S associated with resistance to extended-spectrum cephalosporins [15]. Furthermore, F9o contained the adenine deletion in the inverted repeat sequence of the mtrR promoter, resulting in an overexpression of the MtrCDE efflux pump, and G120K and A121D amino acid substitutions in PorB1b, which further increase ESC MICs and contribute to the MDR phenotype of F9o. The quinolone resistance determining regions (QRDRs) carried S91F and D95A substitutions in GyrA (subcomponent of DNA gyrase) and a S87R substitution in ParC (subcomponent of Topoisomerase IV), which explained the high-level resistance to ciprofloxacin. No tetM gene was detected but the rps/ gene contained a mutation conferring the $V_{57} \mathrm{M}$ amino acid alteration in the $\mathrm{S}_{10}$ ribosomal protein, which contributes to low-level chromosomally-mediated resistance to tetracycline.

\section{Discussion}

In 2010, the first European high-level ceftriaxoneresistant gonococcal isolate was detected in France and characterised in detail [16]. The isolate (F89) harboured a mosaic penA XXXIV allele with an additional $\mathrm{A}_{501} \mathrm{P}$ alteration in $\mathrm{PBP} 2$ and belonged to the internationally spread MDR NG-MAST ST1407 clone [17]. Since 2010, F89 has only been reported in Spain [18] and does not appear to have spread further, which is likely due to a decreased biological fitness of F89 [19]. From 2012 to 2014 , the proportion of NG isolates with resistance to cefixime decreased nearly three-fold from $3.3 \%$ to $1.2 \%$ in France [7].

Here, we report a new MDR NG strain (F9o) with ceftriaxone resistance and intermediate resistance to azithromycin that lead to treatment failure of pharyngeal gonorrhoea with ceftriaxone and doxycycline. F9o differs from the French F89 isolate detected in 2010 [16], however, it is similar to a ceftriaxone-resistant clone initially described in Japan in 2016 (FC428 strain; similar antibiogram and an identical mosaic penA60.001 allele, MLST $_{1903}$, and NG-STAR ${ }_{233}$ ) [14]. The FC428 strain appears to have spread internationally and has been subsequently detected in Australia, Canada and Denmark [20]. In early-2018 the first global strain with ceftriaxone resistance combined with highlevel azithromycin resistance was identified in the UK [21], followed by two similar cases in Australia [22].

Unfortunately, the patient presented in this article and her partner are currently lost for further followup which is of concern as it creates opportunities for spread of this ceftriaxone-resistant strain. 


\section{Conclusion}

An MDR gonococcal strain with ceftriaxone resistance and intermediate resistance to azithromycin was found in France in late-2017. The strain might belong to an internationally spread ceftriaxone-resistant clone, which threatens the recommended dual therapy of gonorrhoea and poses a public health threat. Increased awareness of the spread of ceftriaxone-resistant strains, enhanced antimicrobial resistance surveillance, improved implementation of the recommended dual antimicrobial therapy, partner notification and treatment, and TOC are imperative on an international level. Our treatment failure also illustrates the substantial difficulties in treating pharyngeal gonorrhoea and enhanced focus on detection and treatment is, therefore, crucial. Ultimately, novel therapeutic antimicrobials for gonorrhoea are essential.

\section{Acknowledgements}

This study received financial support from the French National Public Health Agency (Saint Maurice, France) for the French Reference Center of Bacterial STI.

\section{Conflict of interest}

None declared.

\section{References}

1. Wi T, Lahra MM, Ndowa F, Bala M, Dillon JR, Ramon-Pardo P, et al. Antimicrobial resistance in Neisseria gonorrhoeae: Global surveillance and a call for international collaborative action. PLoS Med. 2017;14(7):e1002344. https://doi.org/10.1371/ journal.pmed.1002344 PMID: 28686231

2. World Health Organization (WHO). Global action plan to control the spread and impact of antimicrobial resistance in Neisseria gonorrhoeae. Geneva: WHO; 2012. Available from: http://www. who.int/reproductivehealth/publications/rtis/9789241503501/ en/

3. European Centre for Disease Prevention and Control $(E C D C)$. Response plan to control and manage the threat of multidrugresistant gonorrhoea in Europe. Stockholm: ECDC; 2012. Available from: https://ecdc.europa.eu/sites/ portal/files/media/en/publications/Publications/1206-ECDCMDRgonorrhoea-response-plan.pdf2.

4. Fifer H, Natarajan U, Jones L, Alexander S, Hughes G, Golparian $D$, et al. Failure of dual antimicrobial therapy in treatment of gonorrhea. N Engl J Med. 2016;374(25):2504-6. https://doi. org/10.1056/NEJMC1512757 PMID: 27332921

5. Geisler WM, Uniyal A, Lee JY, Lensing SY, Johnson S, Perry RC, et al. Azithromycin versus Doxycycline for Urogenital Chlamydia trachomatis Infection. N Engl J Med. 2015;373(26):2512-21. https://doi.org/10.1056/NEJMoa1502599 PMID: 26699167

6. European Committee on Antimicrobial Susceptibility Testing (Eucast). Breakpoint tables for interpretation of MICs and zone diameters Version 7.1, 2017. Europe: Eucast; 2017. Available from: http://www.eucast.org/fileadmin/src/media/PDFs/ EUCAST_files/Breakpoint_tables/v_7.1_Breakpoint_Tables.pdf

7. de Curraize C, Kumanski S, Micaëlo M, Fournet N, La Ruche G, Meunier F, et al. Ceftriaxone-resistant Neisseria gonorrhoeae isolates (2010 to 2014) in France characterized by using whole-genome sequencing. Antimicrob Agents Chemother. 2016;60(11):6962-4. https://doi.org/10.1128/AAC.01568-16 PMID: 27600036

8. Bankevich A, Nurk S, Antipov D, Gurevich AA, Dvorkin M, Kulikov AS, et al. SPAdes: a new genome assembly algorithm and its applications to single-cell sequencing. J Comput Biol. 2012;19(5):455-77. https://doi.org/10.1089/cmb.2012.0021 PMID: 22506599

9. Gurevich A, Saveliev V, Vyahhi N, Tesler G. QUAST: quality assessment tool for genome assemblies. Bioinformatics.
2013;29(8):1072-5. https://doi.org/10.1093/bioinformatics/ btto86 PMID: 23422339

10. Vallenet D, Calteau A, Cruveiller S, Gachet M, Lajus A, Josso $A$, et al. MicroScope in 2017: an expanding and evolving integrated resource for community expertise of microbial genomes. Nucleic Acids Res. 2017;45(D1):D517-28. https://doi. org/10.1093/nar/gkw1101 PMID: 27899624

11. Unemo M, Dillon J-AR. Review and international recommendation of methods for typing neisseria gonorrhoeae isolates and their implications for improved knowledge of gonococcal epidemiology, treatment, and biology. Clin Microbiol Rev. 2011;24(3):447-58. https://doi.org/10.1128/ CMR.00040-10 PMID: 21734242

12. Bennett JS, Jolley KA, Sparling PF, Saunders NJ, Hart CA, Feavers IM, et al. Species status of Neisseria gonorrhoeae: evolutionary and epidemiological inferences from multilocus sequence typing. BMC Biol. 2007;5(1):35. https://doi. org/10.1186/1741-7007-5-35 PMID: 17825091

13. Demczuk W, Sidhu S, Unemo M, Whiley DM, Allen VG, Dillon JR, et al. Neisseria gonorrhoeae sequence typing for antimicrobial resistance, a novel antimicrobial resistance multilocus typing scheme for tracking global dissemination of $\mathrm{N}$. gonorrhoeae strains. J Clin Microbiol. 2017;55(5):1454-68. https://doi. org/10.1128/JCM.00100-17 PMID: 28228492

14. Nakayama S, Shimuta K, Furubayashi K, Kawahata T, Unemo M, Ohnishi M. New ceftriaxone- and multidrug-resistant Neisseria gonorrhoeae strain with a novel mosaic penA gene isolated in Japan. Antimicrob Agents Chemother. 2016;60(7):4339-41. https://doi.org/10.1128/AAC.00504-16 PMID: 27067334

15. Unemo M, Shafer WM. Antimicrobial resistance in Neisseria gonorrhoeae in the 21st century: past, evolution, and future. Clin Microbiol Rev. 2014;27(3):587-613. https://doi. org/10.1128/CMR.00010-14 PMID: 24982323

16. Unemo M, Golparian D, Nicholas R, Ohnishi M, Gallay A, Sednaoui P. High-level cefixime- and ceftriaxone-resistant Neisseria gonorrhoeae in France: novel penA mosaic allele in a successful international clone causes treatment failure. Antimicrob Agents Chemother. 2012;56(3):1273-80. https:// doi.org/10.1128/AAC.05760-11 PMID: 22155830

17. Harris SR, Cole MJ, Spiteri G, Sánchez-Busó L, Golparian D, Jacobsson S, et al. Euro-GASP study group. Public health surveillance of multidrug-resistant clones of Neisseria gonorrhoeae in Europe: a genomic survey. Lancet Infect Dis. 2018;S1473-3099(18)30225-1. (Forthcoming). PMID: 29776807

18. Cámara J, Serra J, Ayats J, Bastida T, Carnicer-Pont D, Andreu A, et al. Molecular characterization of two high-level ceftriaxoneresistant Neisseria gonorrhoeae isolates detected in Catalonia, Spain. J Antimicrob Chemother. 2012;67(8):1858-60. https:// doi.org/10.1093/jac/dks162 PMID: 22566592

19. Vincent LR, Kerr SR, Tan Y, Tomberg J, Raterman EL, Dunning Hotopp JC, et al. In vivo-selected compensatory mutations restore the fitness cost of mosaic penA alleles that confe ceftriaxone resistance in Neisseria gonorrhoeae. MBio. 2018;9(2):e01905-17. https://doi.org/10.1128/mBio.01905-17 PMID: 29615507

20. Lahra MM, Martin I, Demczuk W, Jennison AV, Lee K-I, Nakayama S-I, et al. Cooperative recognition of internationally disseminated ceftriaxone-resistant Neisseria gonorrhoeae strain. Emerg Infect Dis. 2018;24(4):735-43. https://doi. org/10.3201/eid2404.171873 PMID: 29553335

21. British Government. Multi-drug resistant gonorrhoea in England: 2018. March 2018. England: British Government; 2018. Available from: https://www.gov.uk/government/ publications/multi-drug-resistant-gonorrhoea-in-england-2018

22. Australian Government Department of Health. Multi-drug resistant gonorrhoea. April 2018. Australia: Department of Health; 2018. Available from: http://www.health.gov.au/ internet/main/publishing.nsf/Content/mr-yr18-dept-depto04. htm

\section{License and copyright}

This is an open-access article distributed under the terms of the Creative Commons Attribution (CC BY 4.0) Licence. You may share and adapt the material, but must give appropriate credit to the source, provide a link to the licence, and indicate if changes were made.

This article is copyright of the authors, 2018. 